\title{
Article
}

\section{Contact Symmetries of a Model in Optimal Investment Theory}

\author{
Daniel J. Arrigo * and Joseph A. Van de Grift
}

Department of Mathematics, University of Central Arkansas, Conway, AR 72035, USA; jvandegrift@cub.uca.edu * Correspondence: darrigo@uca.edu

\begin{abstract}
It is generally known that Lie symmetries of differential equations can lead to a reduction of the governing equation(s), lead to exact solutions of these equations and, in the best case scenario, lead to a linearization of the original equation. In this paper, we consider a model from optimal investment theory where we show the governing equation possesses an extensive contact symmetry and, through this, we show it is linearizable. Several exact solutions are provided including a solution to a particular terminal value problem.
\end{abstract}

Keywords: contact symmetry; optimal investment theory; linearization; exact solutions

Citation: Arrigo, D.J.; Van de Grift, J.A. Contact Symmetries of a Model in Optimal Investment Theory. Symmetry 2021, 13, 217. https:// doi.org/10.3390/sym13020217

\section{Academic Editor: José Carlos R.}

Alcantud

Received: 31 December 2020

Accepted: 26 January 2021

Published: 28 January 2021

Publisher's Note: MDPI stays neutral with regard to jurisdictional clai$\mathrm{ms}$ in published maps and institutional affiliations.

Copyright: (C) 2021 by the authors. Licensee MDPI, Basel, Switzerland. This article is an open access article distributed under the terms and conditions of the Creative Commons Attribution (CC BY) license (https:// creativecommons.org/licenses/by/ $4.0 /)$.

\section{Introduction}

Nonlinear partial differential equations (NLPDEs) play an integral part in describing the world around us. They can be found in the fields of nonlinear diffusion, wave propagation, Mathematical Biology, ray optics, solid mechanics, and financial mathematics to name just a few (see, for example, refs. [1-5] and the references within). However, obtaining exact solutions of these equations is usually a difficult task and techniques for obtaining solutions is a current area of research. One popular technique are symmetry methods probably due to the fact that the method is rather algorithmic and thus computer algebra systems such as Maple and Mathematica can be used. Symmetry methods have been extensively used in a number of fields, and we refer the reader to the books by Arrigo [6], Bluman et al. [7,8], Bordag [9], Cantwell [10], Cherniha et al. [11], and Olver [12] .

In this paper, we are interested in a model from optimal investment theory. Consider an investment portfolio consisting of $n+1$ assets. Let the first asset be a bond and the next $n$ assets be stocks, all of which are traded continuously. In the simplest case where $n=1$, the value of the portfolio, $u(t, x)$, for time $t$ and investment amount $x$, one model presented by see Yong [13] is the NPDE

$$
u_{t}+r x u_{x}-\frac{(b-r) u_{x}^{2}}{2 \sigma u_{x x}}=0,
$$

or

$$
\left(u_{t}+r x u_{x}\right) u_{x x}-\theta u_{x}^{2}=0 .
$$

where $\theta=\frac{b-r}{2 \sigma}$ and the variables $r, b, \sigma$ represent the interest rate, appreciation rate, and volatility, respectively, and are assumed constant with $\sigma>0$ and $b-r>0$.

A classical symmetry analysis was performed by Yang and $\mathrm{Xu}$ [14] who were able to show that (2) admitted the symmetry generator

$$
\Gamma=T \frac{\partial}{\partial t}+X \frac{\partial}{\partial x}+U \frac{\partial}{\partial u},
$$

where

$$
\begin{aligned}
& T=c_{1}, \\
& X=c_{2} x+c_{3} e^{r t}, \\
& U=c_{4} u+c_{5},
\end{aligned}
$$


where $c_{i}, i=1 \ldots 5$ are arbitrary constants. Through their analysis, they were able to exploit several of these symmetries to obtain a number of reductions and, in some cases, construct exact solutions. It is natural to ask whether (2) admits symmetries that are more general than Lie point symmetries. In this paper, we consider contact symmetries of (2), and we will show that, in fact, (2) admits a rather large contact symmetry which leads to its linearization. In addition to recovering known solutions, we will obtain new exact solutions. We also solve a particular terminal value problem.

\section{Contact Symmetries}

In this section, we construct contact symmetries of (2). If we denote this original NLPDE by $\Delta$ so

$$
\Delta=\left(u_{t}+r x u_{x}\right) u_{x x}-\theta u_{x}^{2}=0,
$$

then contact symmetries are given by

$$
\left.\Gamma^{(2)} \Delta\right|_{\Delta=0}=0,
$$

where the infinitesimal generator $\Gamma$ is

$$
\Gamma=U \frac{\partial}{\partial u^{\prime}},
$$

where $U=U\left(t, x, u, u_{t}, u_{x}\right)$; the first and second extensions of the generator are

$$
\begin{aligned}
& \Gamma^{(1)}=\Gamma+D_{t} U \frac{\partial}{\partial u_{t}}+D_{x} U \frac{\partial}{\partial u_{x}}, \\
& \Gamma^{(2)}=\Gamma^{(1)}+D_{t}^{2} U \frac{\partial}{\partial u_{t t}}+D_{t} D_{x} U \frac{\partial}{\partial u_{t x}}+D_{x}^{2} U \frac{\partial}{\partial u_{x x}},
\end{aligned}
$$

where the operators $D_{t}$ and $D_{x}$ are

$$
\begin{aligned}
& D_{t}=\frac{\partial}{\partial t}+u_{t} \frac{\partial}{\partial u}+u_{t t} \frac{\partial}{\partial u_{t}}+u_{t x} \frac{\partial}{\partial u_{x}}+u_{t t t} \frac{\partial}{\partial u_{t t}}+u_{t t x} \frac{\partial}{\partial u_{t x}}+u_{t x x} \frac{\partial}{\partial u_{x x}} \cdots \\
& D_{x}=\frac{\partial}{\partial x}+u_{x} \frac{\partial}{\partial u}+u_{t x} \frac{\partial}{\partial u_{t}}+u_{x x} \frac{\partial}{\partial u_{x}}+u_{t t x} \frac{\partial}{\partial u_{t t}}+u_{t x x} \frac{\partial}{\partial u_{t x}}+u_{x x x} \frac{\partial}{\partial u_{x x}} \cdots
\end{aligned}
$$

This leads to the set of determining equations

$$
\begin{aligned}
& U_{u_{t} u_{t}}=0, \\
& \theta u_{x}^{2} U_{u_{t} u_{x}}+\left(r x u_{x}+u_{t}\right) U_{x u_{t}}+u_{x}\left(r x u_{x}+u_{t}\right) U_{u u_{t}}=0, \\
&\left(u_{t}+r x u_{x}\right)^{2} U_{x x}+2 u_{x}\left(u_{t}+r x u_{x}\right)^{2} U_{x u}+u_{x}^{2}\left(u_{t}+r x u_{x}\right)^{2} U_{u u}+ \\
& 2 \theta u_{x}^{2}\left(u_{t}+r x u_{x}\right) U_{x u_{x}}+2 \theta u_{x}^{3}\left(u_{t}+2 r x u_{x}\right) U_{u u_{x}}+ \\
& \theta^{2} u_{x}^{4} U_{u_{x} u_{x}}-\theta u_{x}\left(2 u_{t}+r x u_{x}\right) U_{x}-r \theta u_{x}^{3} U_{u_{x}}+\theta u_{x}^{2} U_{t}=0 .
\end{aligned}
$$

Although somewhat a laborious calculation, we find the solution of (10) to be 


$$
\begin{aligned}
U & =F\left(t, u_{x}\right)+c_{1} u_{t}+c_{2} x u_{x}+c_{3}\left(x u_{x}-u\right) \\
& +c_{4}\left(-\frac{x u_{x}-u}{2 \theta} \ln u_{x}+\frac{t}{2 \theta}\left((\theta-r) x u_{x}+(\theta+r) u\right)\right) \\
& +c_{5}\left(t u_{t}-\frac{1}{4 \theta}\left((\theta-r) x u_{x}+(\theta+r) u\right) \ln u_{x}+\frac{(\theta+r)^{2}}{4 \theta} t\left(x u_{x}-u\right)\right) \\
& +c_{6}\left(t^{2} u_{t}+\frac{x u_{x}-u}{4 \theta} \ln ^{2} u_{x}-\frac{t}{2 \theta}\left((\theta-r) x u_{x}+(\theta+r) u\right) \ln u_{x}\right. \\
& \left.+\left(\frac{(r+\theta)^{2} t^{2}-2 \theta t}{4 \theta}\right)\left(x u_{x}-u\right)\right)
\end{aligned}
$$

where $c_{i}, i=1 \ldots 6$ are arbitrary constants and the function $F\left(t, u_{x}\right)$ satisfies

$$
\theta u_{x}^{2} F_{u_{x} u_{x}}-r u_{x} F_{u_{x}}+F_{t}=0 .
$$

Equation (12) is linear and possesses an infinite number of solutions, which means that there are an infinite number of symmetries to (2). Furthermore, since there is a particular function $F$ in (11) that satisfies a linear PDE, this suggests that (2) can be transformed to a linear PDE (Bluman and Kumei [15]). As both $t$ and $u_{x}$ are independent variables in (12), this indicates that maybe we should use these as new independent variables.

\section{A Linearization}

Since the symmetry obtained in the last section contains an arbitrary function that satisfies a linear PDE, this suggests that the original PDE is linearizable. Introducing the new variables

$$
t=T, \quad x=U_{X}, \quad u=X U_{X}-U,
$$

where $U=U(T, X)$, derivatives transform as

$$
u_{t}=-U_{T}, \quad u_{x}=X, \quad u_{x x}=\frac{1}{U_{X X}},
$$

and (2) becomes

$$
\theta X^{2} U_{X X}-r X U_{X}+U_{T}=0,
$$

which is exactly (12). Interestingly enough, (15) looks remarkably similar to the Black-ScholesMerton equation $[16,17]$, which is known to be mappable to the linear heat equation $([16,18])$, so it should come as no surprise that the same is true for (15). However, it would make the contact transformation (13) more complicated and, thus, we will not pursue this line any further.

In the next section, we obtain simple exact solutions of (2) in addition to exploiting Lie symmetries of the linearized Equation (15) to obtain additional solutions.

\section{Exact Solutions}

We have shown that the nonlinear PDE

$$
\left(u_{t}+r x u_{x}\right) u_{x x}-\theta u_{x}^{2}=0
$$

can be transformed to the linear PDE

$$
\theta X^{2} U_{X X}-r X U_{X}+U_{T}=0
$$

via the transformation

$$
t=T, \quad x=U_{X}, \quad u=X U_{X}-U .
$$


We are now in a position to obtain a number of exact solutions to (16). For example, (17) admits separable solutions of the form

$$
U=F(T) G(X)
$$

where $F$ and $G$ satisfies

$$
\begin{aligned}
F^{\prime}-k F & =0 \\
\theta X^{2} G^{\prime \prime}-r X G^{\prime}+k G & =0
\end{aligned}
$$

where $k$ is a separation constant. Equation (20a) is easily solved giving

$$
F=F_{0} e^{k T}
$$

for some arbitrary constant $F_{0}$. Equation (20b) possesses solutions of the form $X^{m}$, where $m$ is a solution of

$$
\theta m^{2}-(r+\theta) m+k=0 .
$$

For example, if $k=s m$ (where $s$ is some constant), then the solution of (22) is

$$
m_{1}=0, m_{2}=\frac{r+\theta-s}{\theta},
$$

leading to the exact solution

$$
U=c_{1}+c_{2} X^{m} e^{m T}, \quad m=\frac{r+\theta-s}{\theta}
$$

Passing (24) through the transformation (18) (resetting the constants $c_{1}$ and $c_{2}$ ) gives

$$
u=c_{1}+c_{2} e^{\alpha(s t-\ln x)}, \quad \alpha=\frac{r+\theta-1}{1-r},
$$

which recovers the exact solution presented by Yang and $\mathrm{Xu}$ [14] by choosing $c_{1}=0, c_{2}=1$ and $s=1$.

As a second example, if $k=r$, then, from (22), we obtain $m=1, r / \theta(\neq 1)$ and we obtain the solution to (20b) as

$$
G=g_{1} X+g_{2} X^{r / \theta}
$$

and the general solution to (17)

$$
U=\left(g_{1} X+g_{2} X^{r / \theta}\right) e^{r T} .
$$

Passing (27) through the transformation (18) leads to the exact solution

$$
u=a\left(x+b e^{r t}\right)^{\frac{r}{r-\theta}} e^{-\frac{r \theta}{r-\theta} t}
$$

of (16), which we believe to be new. In the cases where $r=\theta$, the solution of (20b) is

$$
G=g_{1} X+g_{2} X \ln X
$$

and the general solution to (17)

$$
U=\left(c_{1} X+c_{2} X \ln X\right) e^{r T} .
$$

Passing (30) through the transformation (18) leads to the exact solution

$$
u=c_{1} e^{r t+c_{2} x e^{-r t}},
$$


which was given in [14] in the case of $r=\theta$.

Of course, other choices of $m$ that satisfy (22) would lead to exact solutions of (16) which we will not pursue here.

We also note that symmetries of (17) can be used to generate new solutions of (17), which could lead to new solutions of (16). It is well known (Broadbridge and Arrigo [19]) that, if (17) possesses symmetries with the generator

$$
\Gamma=\Sigma \frac{\partial}{\partial T}+\Phi \frac{\partial}{\partial X}+(\Xi U+Q(T, X)) \frac{\partial}{\partial U},
$$

where $\Sigma, \Phi$, and $\Xi$ have some particular forms and $Q$ satisfies the original PDE (17), then, if one has one seed solution, say $U=U_{0}(T, X)$, then additional solutions can be obtained from

$$
Q=\Sigma \frac{\partial U_{0}}{\partial T}+\Phi \frac{\partial U_{0}}{\partial X}-\Xi U_{0}
$$

For example, (17) admits the symmetry generator (32) where $\Sigma, \Phi$, and $\Xi$ are given by

$$
\begin{aligned}
\Sigma & =c_{1}+2 c_{2} T+c_{3} T^{2} \\
\Phi & =\left(\left(c_{2}+c_{3} T\right) \ln X+c_{4}+c_{5} T\right) X \\
\Xi & =\left(\frac{c_{3}}{4 \theta} \ln ^{2} X+\frac{\left(c_{2}+c_{3} T\right)(r+\theta)+c_{5}}{2 \theta} \ln X\right. \\
& \left.+\frac{(r+\theta)^{2}\left(2 c_{2}+c_{3} T\right) T+2 c_{5}(r+\theta) T}{4 \theta}-\frac{c_{3} T}{2}+c_{6}\right) U
\end{aligned}
$$

where $c_{i}, i=1 \ldots 6$ are arbitrary constants.

One particularly simple solution of (17) is

$$
U=1 .
$$

From (33), we obtain the solution

$$
\begin{aligned}
Q=-\left(\frac{c_{3}}{4 \theta}\right. & \ln ^{2} X+\frac{\left(c_{2}+c_{3} T\right)(r+\theta)+c_{5}}{2 \theta} \ln X \\
& \left.+\frac{(r+\theta)^{2}\left(2 c_{2}+c_{3} T\right) T+2 c_{5}(r+\theta) T}{4 \theta}-\frac{c_{3} T}{2}+c_{6}\right) .
\end{aligned}
$$

Passing (36) through the transformation (18) leads to a solution that is parametric in its nature (which we do not list here); however, setting $c_{3}=0$, we obtain (omitting translational constants)

$$
u=\frac{(r+\theta)\left(c_{2}(r+\theta)+c_{5}\right)}{2 \theta} t-\frac{c_{2}(r+\theta)+c_{5}}{\theta} \ln x
$$

which we believe is new. Of course, other seed solutions could lead to an abundance of exact solutions to (17) which, in turn, would lead to exact solutions to (16).

\section{A Particular Terminal Value Problem}

A particular problem of interest is one that is given in Koleva and Vulkow [20], which is to solve (2) subject to the terminal condition

$$
u\left(x, t^{*}\right)=1-e^{-\mu x} .
$$

Here, we introduce a slight variation of (18)

$$
t=T+t^{*}, \quad x=\frac{U_{X}}{\mu}, \quad u=X U_{X}-U+1 .
$$


Under this transformation, the PDE (2) still transforms to (17); however, the terminal condition (38) turns into the initial condition

$$
U(X, 0)=X-X \ln X
$$

At this point, we exploit the symmetries obtained in the previous section. With these symmetries, we associate an invariant surface condition

$$
\Sigma U_{T}+\Phi U_{X}=\Xi U
$$

where $\Sigma, \Phi$ and $\Xi$ are given in (34). From (40) and (17), we obtain the initial conditions

$$
U_{X}(X, 0)=-\ln X, \quad U_{T}(X, 0)=\theta X-r X \ln X .
$$

Requiring that (40) and (42) satisfy (41) on the boundary $T=0$ gives

$$
c_{3}=0, \quad c_{4}=(\theta-r) c_{1}-c_{2}, \quad c_{5}=(\theta-r) c_{2}, \quad c_{6}=\theta c_{1},
$$

where $c_{1}$ and $c_{2}$ are arbitrary. Here, we choose $c_{1}=1, c_{2}=0$, leading to the invariant surface condition

$$
U_{T}+(\theta-r) X U_{X}=\theta U
$$

This is easily solved giving

$$
U=e^{\theta T} F(\ln X+(r-\theta) T) .
$$

Imposing the initial condition (40) on the solution (45) gives

$$
F(\ln X)=X-X \ln X
$$

and, if we let $\lambda=\ln X$, we obtain

$$
F(\lambda)=(1-\lambda) e^{\lambda}
$$

and, from (45), we obtain

$$
U=X(1-\ln X+(\theta-r) T) e^{r T}
$$

and one can indeed verify that (48) does satisfy (17) and (42). As the final step, we pass (48) through the transformation (39), leading to

$$
u=1-e^{-\theta\left(t^{*}-t\right)-\mu x e^{r\left(t^{*}-t\right)},}
$$

which satisfies the original PDE (2) and the terminal condition (38).

\section{Conclusions}

It is well known that classical Lie symmetries can lead to a reduction of a given PDE and sometimes lead to exact solutions of the equation. The best case scenario, albeit rare, indicates that the original equation is linearizable. In this paper, we constructed the contact symmetries of a model from an optimal investment theory, which led to a linearization of the given PDE. Several exact solutions were obtained. The symmetries of the linearized equation were also considered where an additional exact solution was obtained in addition to solving a particular terminal value problem.

Author Contributions: The authors contributed equally to this work. All authors have read and agreed to the published version of the manuscript.

Funding: This research received no external funding. 
Acknowledgments: We thank the reviewers for their valuable comments and suggestions. The second author gratefully acknowledges the support from the University of Central Arkansas in terms of a graduate assistantship.

Conflicts of Interest: The authors declare no conflict of interest.

\section{References}

1. Arrigo, D.J. Analytical Techniques for Solving Nonlinear Partial Differential Equations; Morgan-Claypool: San Rafael, CA, USA, 2019.

2. Debnath, L. Nonlinear Partial Differential Equations for Scientists and Engineers, 3rd ed.; Springer: New York, USA 2012.

3. Logan, J.D. An Introduction to Nonlinear Partial Differential Equations, 2nd ed.; John Wiley \& Sons, Inc.: Hoboken, NJ, USA, 2008.

4. Murray, J.D. Mathematical Biology 1: An Introduction; Springer: Berlin/Heidelberg, Germany, 2002.

5. Murray, J.D. Mathematical Biology II: Spatial Models and Biomedical Applications; Springer: Berlin/Heidelberg, Germany, 2003.

6. Arrigo, D.J. Symmetry Analysis of Differential Equations: An Introduction; John Wiley \& Sons, Inc.: Hoboken, NJ, USA, 2015.

7. Bluman, G.; Kumei, S. Symmetries and Differential Equations; Springer: New York, NY, USA, 1989.

8. Bluman, G.; Anco, S.C. Symmetry and Integration Methods for Differential Equations; Springer: Berlin/Heidelberg, Germany, 2002.

9. Bordag, L.A. Geometrical Properties of Differential Equations. Applications of the Lie Group Analysis in Financial Mathematics; World Scientific: Singapore, 2015.

10. Cantwell, B.J. Introduction to Symmetry Analysis; Cambridge Texts in Applied Mathematics: Cambridge, UK, 2002.

11. Cherniha, R.; Mykola, S.; Pliukhin, O. Nonlinear Reaction-Diffusion-Convection Equations: Lie and Conditional Symmetry, Exact Solutions and Their Applications; CRC Press: Boca Raton, FL, USA, 2018.

12. Olver, P. Applications of Lie Groups to Differential Equations; Springer: New York, NY, USA, 1986.

13. Yong, J. Introduction to mathematical finance. In Mathematical Finance-Theory and Applications; Young, J., Cont, R., Eds.; Higher Education Press: Bejing, China, 2000.

14. Yang, S.; Xu, T. Lie symmetry analysis for a parabolic Monge-Ampere equation in the optimal investment theory. J. Comput. Appl. Math. 2019, 346 483-489. [CrossRef]

15. Bluman, G.; Kumei, S. When nonlinear differential equations are equivalent to linear differential equations. SIAM J. Appl. Math. 1982, 42, 1157-1173.

16. Merton, R.C. Theory of Rational Option Pricing. Bell J. Econ. Manag. Sci. 1973, 4, 141-183. [CrossRef]

17. Black, F.; Scholes, M. The pricing of Options and Corporate Liabilities. J. Political Econ. 1973, 81, 637-654. [CrossRef]

18. Gazizov, R.K.; Ibragimov, N.H. Lie Symmetry Analysis of Differential Equations in Finance. Nonlinear Dyn. 1998, 17, 387-407. [CrossRef]

19. Broadbridge, P.; Arrigo, D.J. All solutions of standard symmetric linear partial differential equations have a classical Lie symmetry. J. Math. Anal. Appl. 1999, 234, 109-122. [CrossRef]

20. Koleva, M.N.; Vulkov, L.G. A numerical study of a parabolic Monge-Ampere equation in mathematical finance. In Numerical Methods and Applications. Lecture Notes in Computer Science; Dimov, I., Dimova, S., Kolkovska, N., Eds.; Springer: Berlin/Heidelberg, Germany, 2010; Volume 6046. 